Case Report

\title{
The Histopathological Finding of the Surgically Extracted Atypical Dome-Shaped Choroidal Osteoma
}

\author{
Hirona Bessho, ${ }^{1}$ Hisanori Imai, ${ }^{1,2}$ and Atsushi Azumi ${ }^{1}$ \\ ${ }^{1}$ Department of Ophthalmology, Kobe Kaisei Hospital, Kobe 657-0068, Japan \\ ${ }^{2}$ Department of Organ Therapeutics, Division of Ophthalmology, Kobe University Graduate School of Medicine, Kobe 650-0017, Japan
}

Correspondence should be addressed to Hisanori Imai; hisimai@med.kobe-u.ac.jp

Received 4 January 2017; Accepted 8 March 2017; Published 15 March 2017

Academic Editor: Alexander A. Bialasiewicz

Copyright (C) 2017 Hirona Bessho et al. This is an open access article distributed under the Creative Commons Attribution License, which permits unrestricted use, distribution, and reproduction in any medium, provided the original work is properly cited.

\begin{abstract}
Purpose. To report a case of atypical dome-shaped choroidal osteoma, which was diagnosed by histopathological finding of surgically extracted tumor. Case Report. A 35-year-old woman presented with visual field abnormality in the left eye (OS). Her best-corrected visual acuity with Landolt ring chart was 1.0 OS. The funduscopic examination revealed a yellowish dome-shaped choroidal tumor located in the temporal side of the macula with exudative retinal detachment. 25-gauge pars plana vitrectomy and the extraction of the tumor were performed for the definitive diagnosis. Results. As a result of histopathological finding from the extracted tumor, she was diagnosed with choroidal osteoma. 10 months after the last surgery, the BCVA is 0.7 OS. The tumor is not relapsed. Conclusions. We must keep in mind that choroidal osteoma can be one of the differential diagnoses for the dome-shaped choroidal tumor.
\end{abstract}

\section{Introduction}

Choroidal osteoma is a rare benign intraocular tumor characterized by heterotopic bone of the choroid. Typical choroidal osteoma is slightly elevated from the peripapillary or macular choroid [1-5]. Usually, its characteristic funduscopic, computed tomography (CT), and B-scan ultrasonography findings are sufficient for a clinical diagnosis [6]. However, we face a particular diagnostic problem in case that the clinical and imaging findings are atypical because the biopsy and extraction of this tumor for definitive histopathological diagnosis are considerably difficult [6-8].

Here, we report a case of atypical dome-shaped choroidal osteoma diagnosed by a histopathological finding from the surgically extracted tumor tissue.

\section{Case Report}

A 35-year-old woman was referred to us with visual field abnormality in the left eye (OS). On examination, bestcorrected visual acuity (BCVA) with Landolt ring chart was 1.0 in both eyes at the initial visit. The slit-lamp examination of the anterior segment was normal OS. A fundus examination revealed a yellowish dome-shaped choroidal tumor of approximately 4-disc diameters in size located to the temporal side of the macula with exudative retinal detachment OS (Figures 1(a) and 1(d)). The right-eye findings were unremarkable. Fluorescein and indocyanine angiography revealed the pooling of the dye into the tumor (Figures 1(b) and 1(c)). The tumor was depicted as high intensity on both T1-weighted (Figure 2(a)) and gadolinium enhanced (Figure 2(c)) magnetic resonance images (MRI), and low intensity on short-T1 inversion recovery (STIR) MRI (Figure 2(b)), with maximal dimensions of $5.3 \times 5.7$ $\times 6.0 \mathrm{~mm}$. The tumor was depicted as high density lesion on CT (Figure 2(d)). B-scan ultrasonography showed high internal reflectivity and acoustic shadowing (Figure 2(e)). NIsopropyl-p-123I-iodoamphetamine single photon emission computed tomography (SPECT) obtained at 24 hours after intravenous administration of 123I-IMP showed no positive area. The serum 5-S-cysteinyldopa (5-S-CD) level was high at $9.5 \mathrm{nmol} / \mathrm{l}$ (normal range $1.5-8.0 \mathrm{nmol} / \mathrm{l}$ ). The findings of fundus, MRI, and SPECT and the value of serum 5-S-CD are compatible with an amelanotic malignant melanoma, but the results of other examinations suggested the tumor which is possibly calcified. For a confident diagnosis, we performed 


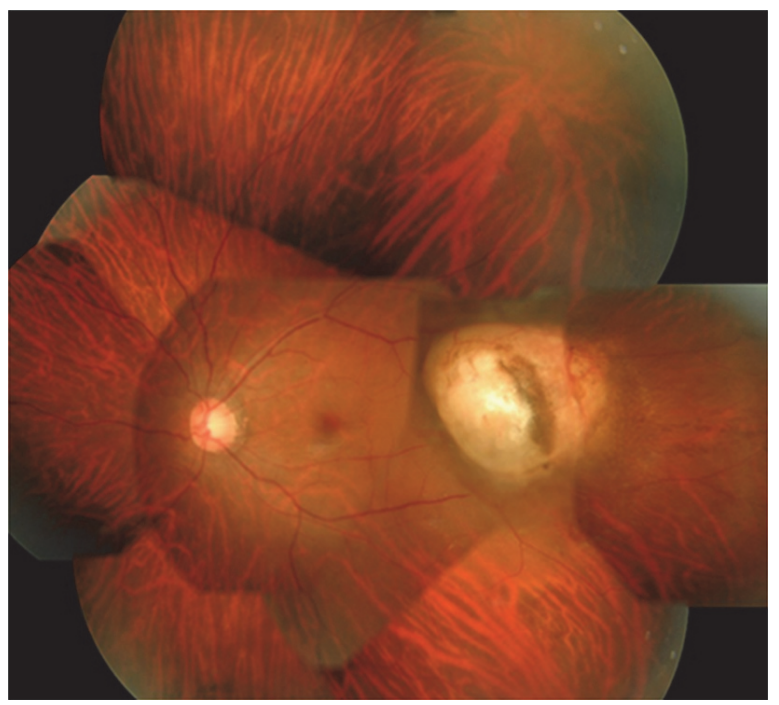

(a)

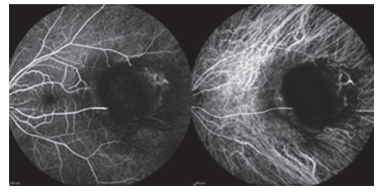

(b)

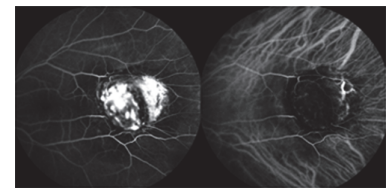

(c)

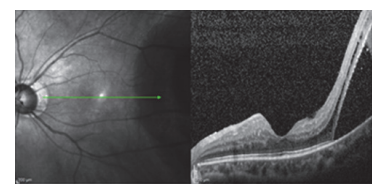

(d)

Figure 1: (a) A fundus examination revealed a yellowish dome-shaped choroidal tumor. (b) Early phase of the fluorescein (left) and indocyanine (right) angiography. (c) Late phase of the fluorescein (left) and indocyanine (right) angiography. The findings of angiographies revealed the pooling of the dye into the tumor. (d) The OCT finding of exudative retinal detachment surround the tumor.

the extraction of the tumor under the written informed consent from the patient and her father. The procedure of the primary operation was listed below. Additional pars plana vitrectomy (PPV) for silicone oil removal and for epiretinal membrane removal and intraocular lens implantation were performed one and three months after the primary operation, respectively. 10 months after the last surgery, the BCVA is 0.7 OS. Choroidal osteoma is not relapsed (Figure 3).

\section{Surgical Technique for the Primary PPV}

Initially, we tried a diagnostic biopsy for the tumor using the standard 25-gauge three-port PPV.

But the biopsy was failed because the tumor was calcified and therefore unresectable. As the next best thing, we performed the extraction of the tumor. Briefly, retinectomy at the surface of the tumor was done after total vitrectomy was performed. 20-gauge pars plana sclerotomies were made by 20-gauge V-lances at 3 and 9 oclock position. The tumor was chipped off from sclera by the V-lances and a
25 -gauge intraocular scissor. The phacoemulsification was performed from $2.4 \mathrm{~mm}$ scleral tunnel and then the lens capsule was grasped and removed by 23 -gauge capsulorhexis forceps from the corneal side port. The scleral tunnel was extended to $10 \mathrm{~mm}$ incision and the tumor was removed from this site. The vitreous cavity was filled with silicone oil at the end of the operation. The surgically excised tumor was fixed with $10 \%$ formalin until use (Supplemental Digital Content in Supplementary Material available online at https://doi.org/10.1155/2017/2874823).

\section{Histopathological Analysis}

Several types of immunohistochemical staining were performed under the patient's writing informed consent. Hematoxylin-eosin staining highlighted the bone formation and hyalinization without osteoblasts (Figures 4(a), 4(b), and 4(c)). Several spindle cells infiltrated around the bone formation. Several pigment-having mononuclear cells were intermingled, suggesting retinal pigment epithelium. 


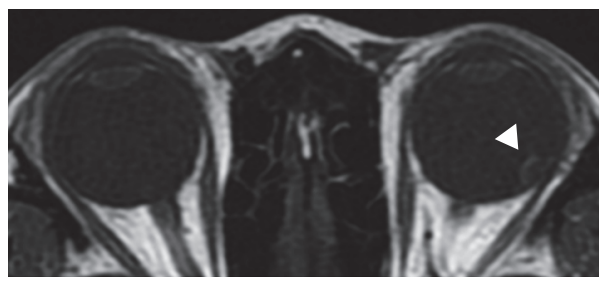

(a)

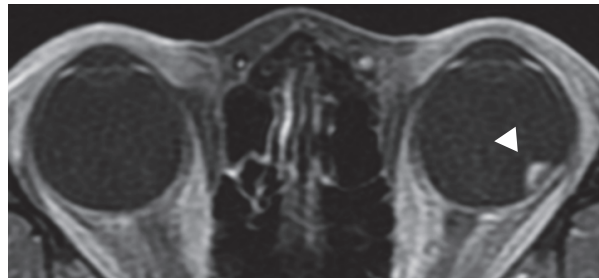

(c)

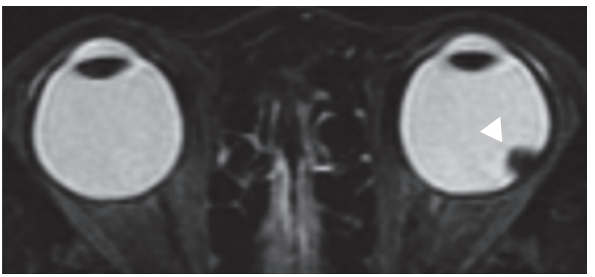

(b)

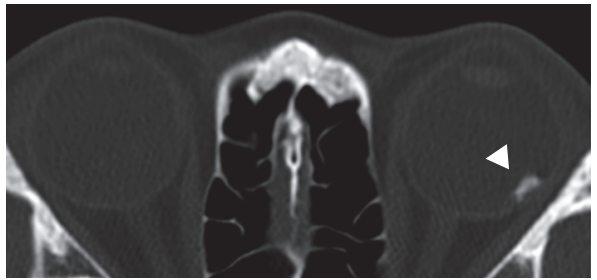

(d)

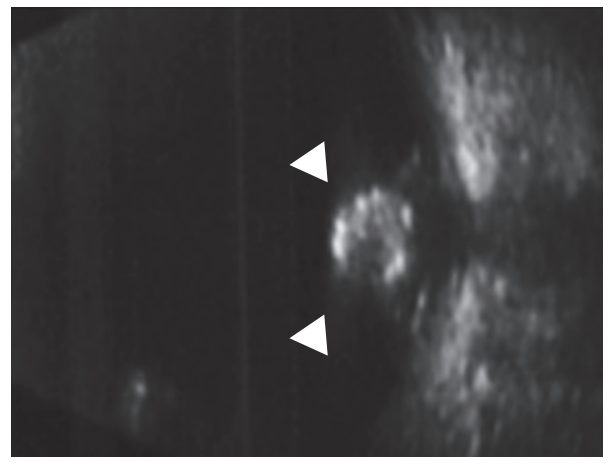

(e)

FIGURE 2: The tumor was depicted as high intensity on both T1-weighted (a) and gadolinium enhanced (c) MRI, low intensity on STIR MRI (b), and high density lesion on CT (d). The B-scan ultrasonography showed high internal reflectivity and acoustic shadowing (e) (arrow heads).

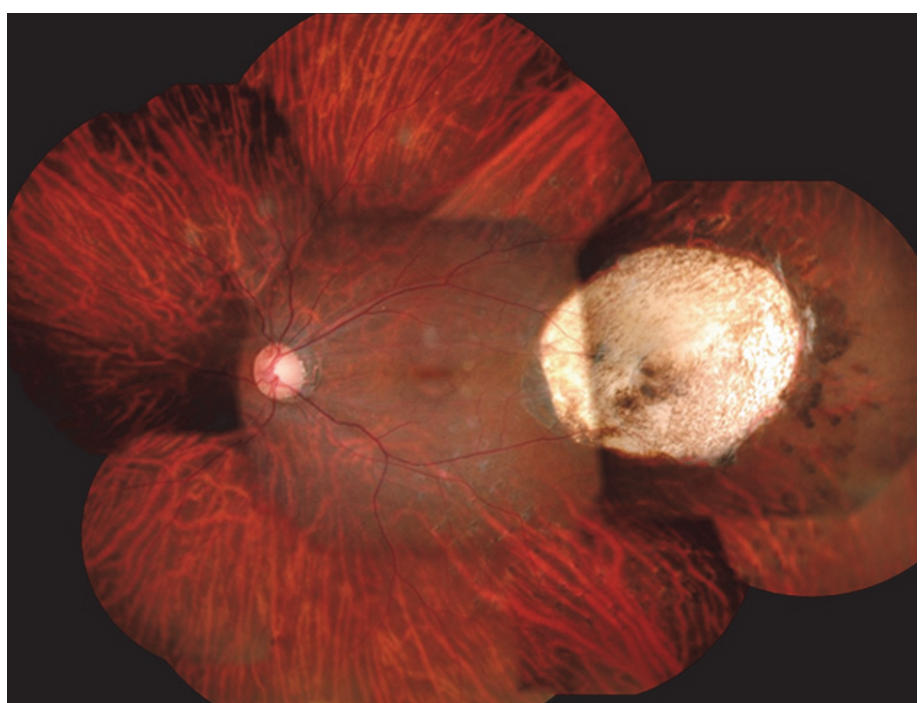

Figure 3: A fundus examination 10 months after the primary operation revealed bare sclera at the temporal side of the macula where the tumor was located. 


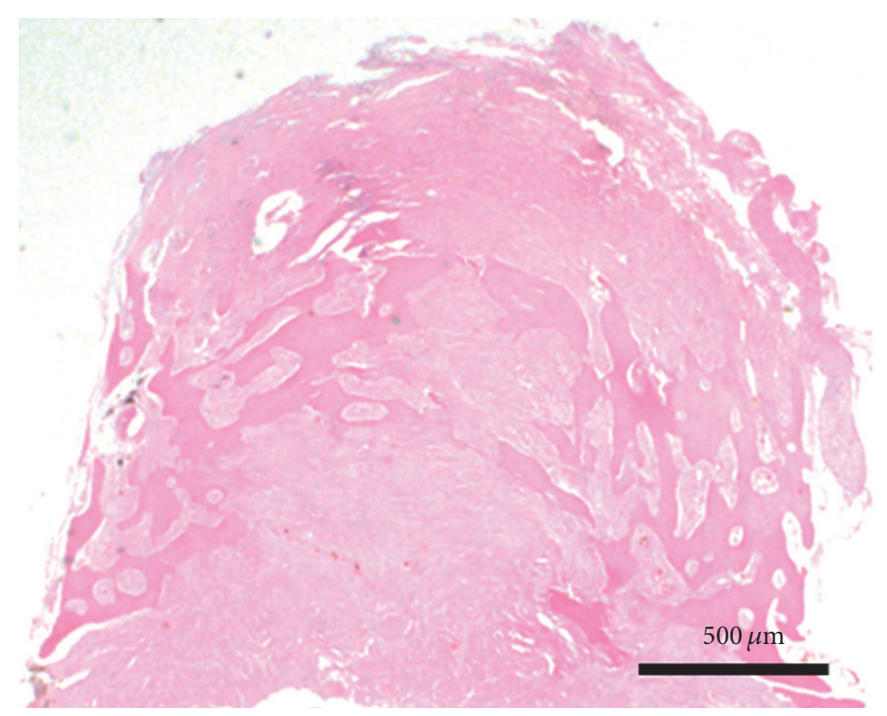

(a)

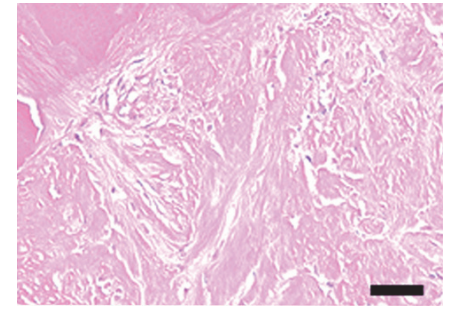

(b)

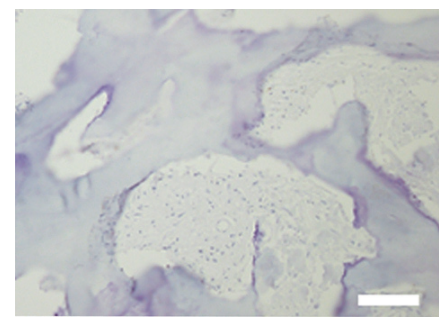

(d)

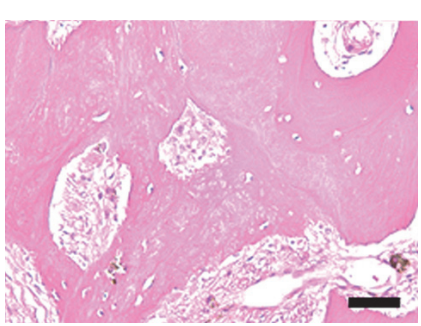

(c)

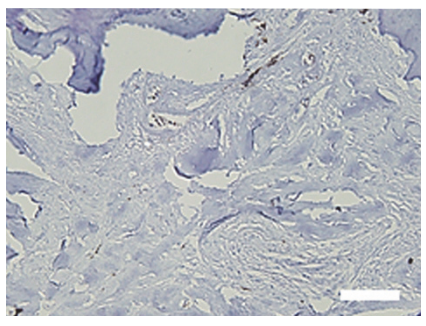

(e)

FIGURE 4: The histopathological finding of the surgically extracted tumor. (a) Hematoxylin-eosin staining of the whole image of extracted tumor. Magnified picture of part of (a) shows hyalinization (b) and bone formation (c). The immunostaining for epithelial membrane antigen (d) and glial fibrillary acidic protein (e) was negative (scale bar: black bar $=20 \mu \mathrm{m}$; white bar $=50 \mu \mathrm{m}$ ).

The immunostaining for epithelial membrane antigen (Figure $4(\mathrm{~d})$ ) and glial fibrillary acidic protein (Figure $4(\mathrm{e})$ ) was negative. From these findings, we diagnosed this tumor as a choroidal osteoma.

\section{Discussion}

In our case, the clinical feature of the tumor was atypical for all differential diagnoses, including choroidal osteoma and amelanotic malignant melanoma, so it was extremely challenging to make a clinical diagnosis as choroidal osteoma using conventional noninvasive examinations. Additional histopathological confirmation of the diagnosis was desirable for differentiation of this calcified tumor from amelanotic malignant melanoma, since the management of intraocular tumors includes different treatment options depending on the pathogenesis [9].

Various intraocular biopsy methods have been proposed with different success rates and side effects [10-16]. In our case, we reluctantly performed the extraction of the tumor because the biopsy was difficult. As a result, we could make a definitive diagnosis and avoid unnecessary invasive treatments including enucleation, radiotherapy, and chemotherapy. However, we eventually needed two additional operations to maintain her visual function. So, we emphasize that the extraction of the tumor could be one of the considerable options when the conclusive diagnosis is needed, but these kinds of invasive techniques should be carefully performed 
because of concerns for iatrogenic vision-threatening ocular complications or extraocular seeding of tumor cells following tumor sampling [9, 13-18].

The histopathological finding of the choroidal osteoma from the living eye is extremely rare, because the morphological confirmation was typically performed after enucleation of the eye. In our case, we finally narrowed down the differential diagnosis to choroidal osteoma, retinal pigment epithelium metaplasia, and retinal astrocytoma. We excluded retinal pigment epithelium metaplasia and retinal astrocytoma because the immunostaining for epithelial membrane antigen and glial fibrillary acidic protein was negative, respectively. We believe that histopathological finding in our case could be one of the important references for the differential diagnosis of the atypical choroidal tumor confused with other entities with similar presentations.

In conclusion, we must keep in mind that choroidal osteoma can be one of the differential diagnoses for atypical dome-shaped yellowish choroidal tumor.

\section{Consent}

Written informed consent from the patient and the approval of the institutional review board in Kobe Kaisei Hospital were obtained for publication of this case report and any accompanying images.

\section{Disclosure}

The authors alone are responsible for the content and writing of the paper.

\section{Conflicts of Interest}

The authors declare that they have no conflicts of interest.

\section{References}

[1] J. D. M. Gass, R. K. Guerry, R. L. Jack, and G. Harris, "Choroidal osteoma," Archives of Ophthalmology, vol. 96, no. 3, pp. 428-435, 1978.

[2] A. T. Williams, R. L. Font, H. J. L. Van Dyk, and F. T. Riekhof, "Osseous choristoma of the choroid simulating a choroidal melanoma: association with a positive 32P test," Archives of Ophthalmology, vol. 96, no. 10, pp. 1874-1877, 1978.

[3] L. Joffe, J. A. Shields, and J. R. Fitzgerald, "Osseous choristoma of the choroid," Archives of Ophthalmology, vol. 96, no. 10, pp. 1809-1812, 1978.

[4] C. L. Shields, J. A. Shields, and J. J. Augsburger, "Choroidal osteoma," Survey of Ophthalmology, vol. 33, no. 1, pp. 17-27, 1988.

[5] C. L. Shields, H. Sun, H. Demirci, and J. A. Shields, "Factors predictive of tumor growth, tumor decalcification, choroidal neovascularization, and visual outcome in 74 eyes with choroidal osteoma," Archives of Ophthalmology, vol. 123, no. 12, pp. 16581666, 2005.

[6] R. M. Alameddine, A. M. Mansour, and E. Kahtani, "Review of choroidal osteomas," Middle East African Journal of Ophthalmology, vol. 21, no. 3, pp. 244-250, 2014.
[7] G. Cennamo, G. Jaccarino, G. de Crecchio, and G. Liguori, "Choroidal osteoma (osseous choristoma): an atypical case," British Journal of Ophthalmology, vol. 74, no. 11, pp. 700-701, 1990.

[8] D. J. Browning, "Choroidal osteoma: observations from a community setting," Ophthalmology, vol. 110, no. 7, pp. 13271334, 2003.

[9] J. Sen, C. Groenewald, P. S. Hiscott, P. A. Smith, and B. E. Damato, "Transretinal choroidal tumor biopsy with a 25-gauge vitrector," Ophthalmology, vol. 113, no. 6, pp. 1028-1031, 2006.

[10] A. Kvanta, S. Seregard, E. D. Kopp, C. All-Ericsson, I. Landau, and L. Berglin, "Choroidal biopsies for intraocular tumors of indeterminate origin," American Journal of Ophthalmology, vol. 140, no. 6, pp. 1002-1006, 2005.

[11] C. L. Shields, M. E. Manquez, H. Ehya, A. Mashayekhi, C. J. Danzig, and J. A. Shields, "Fine-needle aspiration biopsy of iris tumors in 100 consecutive cases. Technique and complications," Ophthalmology, vol. 113, no. 11, pp. 2080-2086, 2006.

[12] M. Bagger, J. F. Tebering, and J. F. Kiilgaard, "The ocular consequences and applicability of minimally invasive 25-gauge transvitreal retinochoroidal biopsy," Ophthalmology, vol. 120, no. 12, pp. 2565-2572, 2013.

[13] S. Seregard, C. All-Ericsson, L. Hjelmqvist, L. Berglin, and A. Kvanta, "Diagnostic incisional biopsies in clinically indeterminate choroidal tumours," Eye (Basingstoke), vol. 27, no. 2, pp. 115-118, 2013.

[14] N. E. Bechrakis, M. H. Foerster, and N. Bornfeld, "Biopsy in indeterminate intraocular tumors," Ophthalmology, vol. 109, no. 2, pp. 235-242, 2002.

[15] N. Abi-Ayad, J.-D. Grange, M. Salle, and L. Kodjikian, “Transretinal uveal melanoma biopsy with 25-gauge vitrectomy system," Acta Ophthalmologica, vol. 91, no. 3, pp. 279-281, 2013.

[16] T. A. McCannel, M. Y. Chang, and B. L. Burgess, "Multiyear follow-up of fine-needle aspiration biopsy in choroidal melanoma," Ophthalmology, vol. 119, no. 3, pp. 606-610, 2012.

[17] A. S. Bansal, C. G. Bianciotto, J. I. Maguire, C. D. Regillo, J. A. Shields, and C. L. Shields, "Safety of pars plana vitrectomy in eyes with plaque-irradiated posterior uveal melanoma," Archives of Ophthalmology, vol. 130, no. 10, pp. 1285-1290, 2012.

[18] A. Grixti, M. Angi, B. E. Damato et al., "Vitreoretinal surgery for complications of choroidal tumor biopsy," Ophthalmology, vol. 121, no. 12, pp. 2482-2488, 2014. 


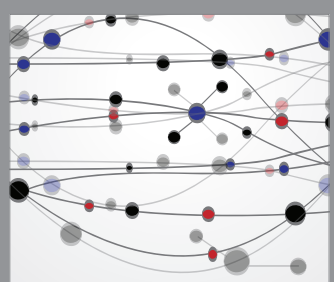

The Scientific World Journal
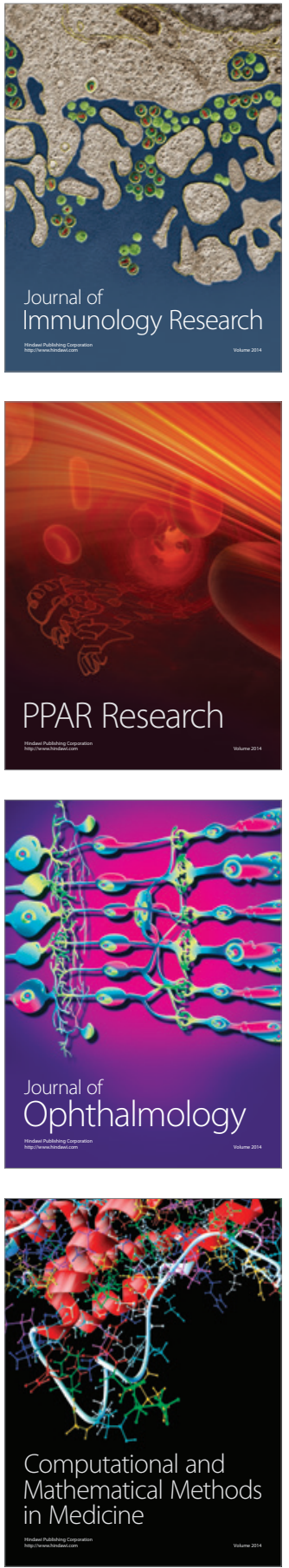

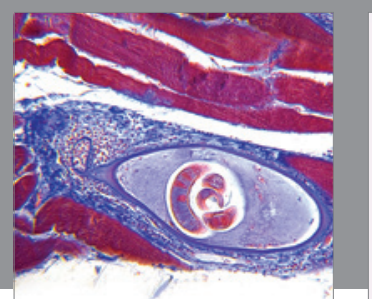

Gastroenterology Research and Practice
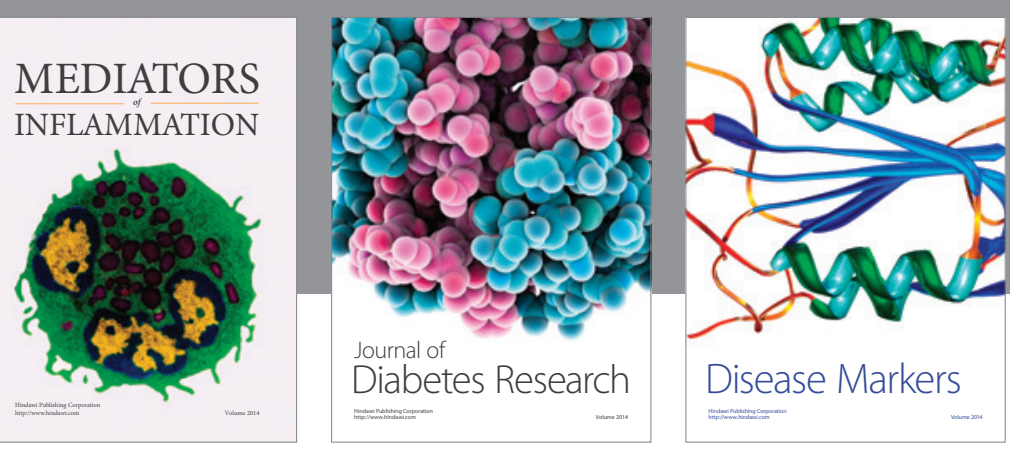

Disease Markers

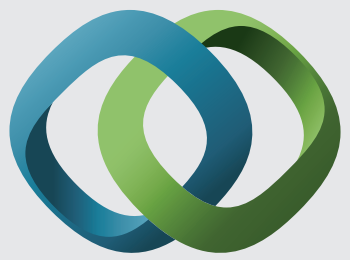

\section{Hindawi}

Submit your manuscripts at

https://www.hindawi.com
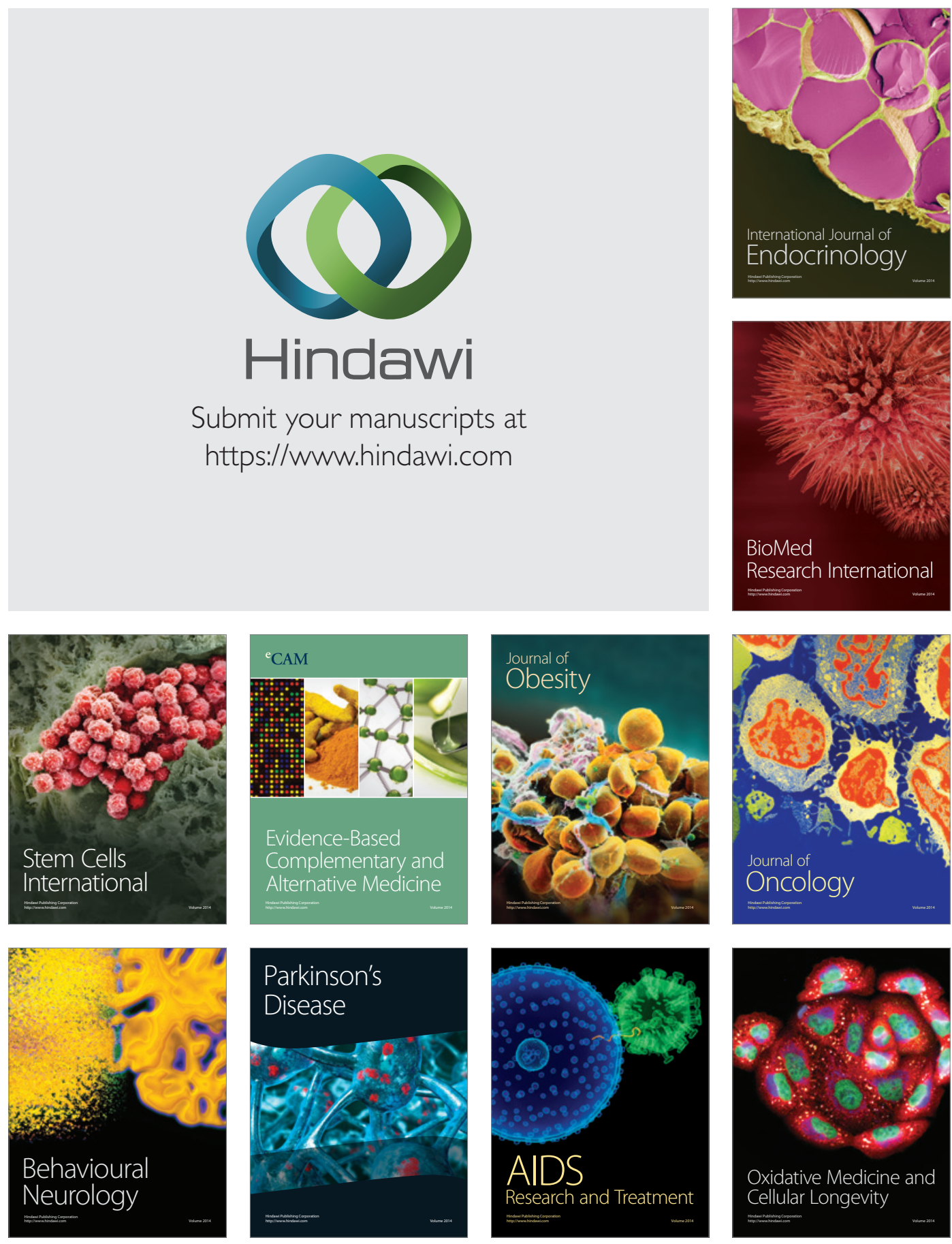